\title{
Performance Measurement Model in the G-PM Tool`
}

\author{
Roland Wismüller ${ }^{1}$, Marian Bubak ${ }^{2,3}$, Włodzimierz Funika ${ }^{2,3}$, \\ Tomasz Arodź ${ }^{2,3}$, and Marcin Kurdziel ${ }^{2,3}$
}

1 LRR-TUM, Institut für Informatik, Technische Universität München, D-85747 Garching, Germany

wismuell@in.tum.de

2 Institute of Computer Science, AGH, al. Mickiewicza 30, 30-059 Kraków, Poland

3 Academic Computer Centre - CYFRONET, Nawojki 11, 30-950 Kraków, Poland \{bubak, funika\}@uci.agh.edu.pl

phone: (+48 12) 61739 64, fax: (+48 12) 63380 54, phone: (+49 89) 28917676

\begin{abstract}
This paper focuses on the model of the performance analysis of distributed grid-enabled applications within the G-PM tool. The major focus is at the issues that arises as a consequence of the on-line application monitoring paradigm the G-PM follows. In particular, two major issues are presented - the consequences of a discrete nature of measuring the function based performance quantities, and the aggregation of performance values that were measured at distant locations, and thus may be desynchronized.
\end{abstract}

Keywords: Grid, performance analysis, performance monitoring

\section{Introduction}

The introduction of grid computing has a great impact on the development of parallel applications. In order to meet the new requirements of the users, the application development tools also have to be adapted to the new computing paradigm.

In this paper, we describe the evolution of one these tools, i.e., the performance analysis tool for grid applications - G-PM [3]. The G-PM is developed as part of the CrossGrid project [4. The tool uses the OCM-G [1] as a low level monitoring system and was designed to allow for on-line performance analysis. During its development, a number of issues concerning the implementation of measurements have to be addressed in order to meet the constraints of the grid computing. This resulted in a relaxation of the strict on-line scheme of the tool. Furthermore, the initially assumed pure pull model of communicating with the OCM-G was augmented to include reservation requests.

* This work was partly funded by the European Commission, project IST-2001-32243, CrossGrid 


\section{Issues with Measuring the Function-Based Metrics}

The G-PM is mainly focused on measuring various quantities (metrics) related to function calls. This may be e.g. duration of the MPI_Send() calls since the application start-up or the amount of data received with MPI_Recv() function. The appropriate library functions are instrumented, so the values of these metrics are gathered at the start and the end of the individual function calls.

Consider the metric measuring the total time spent in MPI_Send() since some start time, denoted as a function $R(t)$ of time $t$. It is not possible to measure the value of $R$ directly. What can be measured is the function $V(t)$, i.e. the total wall-clock time since the beginning of the measurement (in such a case, $V(t)=t)$. The value of $R$ can then be obtained in the following way: $R(t)=\sum_{i: t_{i}^{b}, t_{i}^{e} \leq t}\left(V\left(t_{i}^{e}\right)-V\left(t_{i}^{b}\right)\right)$. The $t_{i}^{b}$ and $t_{i}^{e}$ are the time stamps of the $i$ th begin and end of the function call. In the same approach, the $V(t)$ may, e.g., denote the total amount of data received by $M P I_{-} \operatorname{Recv}()$ until the time $t$.

The G-PM tool inspects the value of $R$ at fixed intervals, i.e., at moments $t_{j}$. The scheme outlined above works fine, as long as $t_{j}$ falls into the range $\left(t_{i}^{e}, t_{i+1}^{b}\right)$ and not in $\left(t_{i}^{b}, t_{i}^{e}\right)$, i.e. falls between consecutive function calls. In the latter case, the measured value of $R$ does not reflect the real value of the metric. This is because the value of $R$ is not updated until the end of the function call. Only then $V\left(t_{i}^{e}\right)$ is known, and difference between the values of $V$ at the end and beginning of the function call (i.e. difference between $V\left(t_{i}^{e}\right) V\left(t_{i}^{b}\right)$ ) can be added to the previous value of $R$. For example, in case of the MPI_Send() delay, the value of $R$, if inspected during the call to MPI_Send(), does not take into account the time spent in this call.

We propose two solutions to this problem. In case the value of $V(t)$ can be accessed at any moment in time, as it is in the case of $V(t)=t$, we modify the function $R$ if the time $t_{j}$ of the query falls within some function call. The current value of $V$ at time $t_{j}$ of the data query (i.e. $V\left(t_{j}\right)$ ) is used instead of the yet unknown value $V\left(t_{i}^{e}\right)$ at yet unknown time $t_{i}^{e}$ of the end of the call.

The scheme outlined above is not suitable if the value of $V(t)$ cannot be queried at any time. That is the case of e.g. MPI_Recv() total data volume transferred. The amount of the data received can be known only at the times of the end of the function call. Therefore, if $R$ is inspected during the call to MPI_Recv(), the value $R\left(t_{j}\right)$ gives only the amount of data received before the beginning of the call. The data received between the beginning of the function call and the querying of the value of $R$ is not taken into account. Thus, the values of the metrics can be misleading, especially when the duration of the function calls is long.

A solution to the problem is to make use of the asynchronous nature of the underlying OCM-G monitoring system. The replies to the data requests are sent to the G-PM asynchronously. Originally, the reply is sent immediately as the data is gathered from the sites of the grid where the monitored application is running. However, this reply can be postponed until the current function call ends, and the value of $R$ reflects the real value. In this way, the G-PM tool 
will always display correct data. However, the visualization may be temporarily delayed, as the replies will no longer arrive immediately after the requests for measurement data to be displayed.

\section{Performance Measurement under Large Network Latency Conditions}

The G-PM makes it possible to narrow the measurement of performance properties of the application to any set of processes specified by the user. The partial results from this locations can be aggregated to produce the final performance measurement value. This is a strong advantage of the tool that facilitates to find performance bottlenecks in the application. On the other hand, the aggregated value is only meaningful, if the measurement was performed on all nodes at the same time interval. Consequently, the monitoring must be synchronized across grid nodes. Currently, the G-PM communicates with the OCM-G in a purely pull model. Each time when a new performance value is needed the requests from the G-PM are sent to OCM-G service manager and then broadcast to local monitors. This preserves the G-PM user's workstation from being overloaded by messages from the OCM-G. However, if the network latency is high and varies significantly across links, the pooling may make the required synchronization impossible.

For this reasons, a hybrid model that shares some properties of both pull and push approaches, is being considered to replace the current communication scheme between G-PM and the OCMG-G. In this hybrid approach, the G-PM asks the OCM-G to measure a performance property at given time intervals. This request is time stamped in OCM-G's service manager and broadcast to local monitors. The local monitors compute the results at requested time intervals and store them in a temporal buffer. Provided that the clocks on local nodes are synchronized (which can be assured within a reasonable accuracy), the time stamp of the initial G-PM request will allow to synchronize the performance measurement across the nodes. To preserve the user workstation from being overloaded, the results are sent from the OCM-G buffers only at an explicit request from the G-PM. An important issue that must be solved in this approach is handling the OCM-G buffers overflow. In this case, the OCM-G may simply perform a partial aggregation of the results, (e.g. via averaging) to reduce the number of data points.

The synchronization of performance measurement across nodes has an additional advantage. Suppose, that the user monitors two performance properties: a mean value of the communication send volume per one second and a total communication send volume from the application startup. Both this performance properties are based on the metric that measures the send volume in communication operations. The desynchronization across the nodes may result in the measurements being performed at different points in time. Consequently, if the synchronization is kept, the values of the metric can be shared. 


\section{Conclusions}

The G-PM tool measures the performance of the distributed application in an on-line fashion. This is a strong advantage in the grid computing where long running applications are common. On the other hand, a number of issues arise which result from the requirements posed by the on-line analysis. Some of these, like e.g., the influence of a discrete nature of the performance monitoring of the function based quantities on the measurement results, are already addressed within the G-PM. Others, like e.g. meaningful aggregation of measurement results from different nodes, still require further study.

An illustration of the evolution of the performance measurement model the G-PM follows is the communication schema between the tool and underlying monitoring layer - OCM-G. Originally, the communication was designed in a strictly pull model. The rationale behind this was the minimization of the probability that the user workstation will be overloaded by performance measurement data. Currently, a new schema is being worked out that is based on a hybrid pull and push model. This schema is believed to still prevent the G-PM from being overloaded, while enabling performance measurements of the application in an on-line, grid-wide synchronized fashion.

\section{References}

1. Baliś, B., Bubak, M., Funika, W., Szepieniec, T., and Wismüller, R.: An Infrastructure for Grid Application Monitoring. In: Kranzlmüller, D. et al. (Eds.), Recent Advances in Parallel Virtual Machine and Message Passing Interface, 9th European PVM/MPI Users' Group Meeting, Sept. - Oct. 2002, Linz, Austria, Lecture Notes in Computer Science 2474, pp. 41-49, Springer-Verlag, 2002.

2. Baliś, B., Bubak, M., Funika, W., Szepieniec, T., and Wismüller, R.: Monitoring and Performance Analysis of Grid Application. In: P.M.A. Sloot et al. (Eds.), Computational Science - ICCS 2003, June 2003, St. Petersburg, Russia, Lecture Notes in Computer Science 2657, pp. 214-224, Springer-Verlag, 2003.

3. Bubak, M., Funika, W., Wismüller, R., Arodz, T., and Kurdziel, M.: The G-PM Tool for Grid-oriented Performance Analysis. In: 1st European Across Grids Conference, Santiago de Compostela, Spain, Feb. 2003.

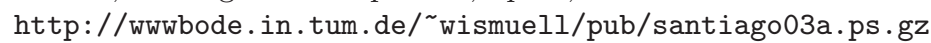

4. CrossGrid - Development of Grid Environment for interactive Applications, EU Project, IST-2001-32243, Technical Annex. http://www.eu-crossgrid.org

5. Wismüller, R., Bubak, M., Funika, W., Arodź, T., and Kurdziel, M.: Support for User-Defined Metrics in the Online Performance Analysis Tool G-PM. Accepted to 2nd European AcrossGrids Conference, Nicosia, Cyprus 2004.

6. Wismüller, R., Bubak, M., Funika, W., and Baliś, B.: A Performance Analysis Tool for Interactive Applications on the Grid. In: Performance Analysis and Grid Computing, Proc. Workshop on Clusters and Computational Grids for Scientific Computing, Sept. 2002, Le Chateau de Faberges de la Tour, France. Kluwer, 2003. In print.

7. Wismüller, R. , Oberhuber, M. , Krammer, J. , and Hansen,O. : Interactive debugging and performance analysis of massively parallel applications. In: Parallel Computing, 22(3):415-442, March 1996. 